\title{
Diffuse aneurysm of the thoracic aorta involving a right aberrant subclavian artery: A three-stage approach
}

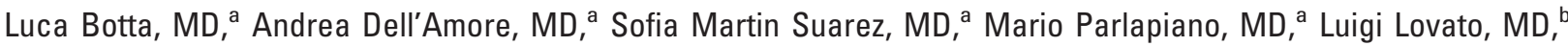 \\ Rossella Fattori, MD, and Roberto Di Bartolomeo, MD, ${ }^{\mathrm{a}}$ Bologna, Italy
}

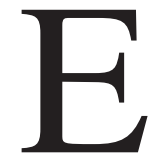

xtensive thoracic aortic aneurysms that involve ascending, arch, and descending segments require challenging repairs associated with substantial morbidity and mortality. Since 1983, staged repair with the elephant trunk technique (ETT) has been the standard procedure for managing these aneurysms. ${ }^{1}$ The aberrant right subclavian artery (ARSA) is a rare entity with a reported prevalence as high as $2 \%$. Aneurysms of the ARSA can cause serious complications. They require surgical treatment, but surgical strategies are still unclear. ${ }^{2}$ In this report, we propose a 3 -stage approach for this complex entity.

\section{Clinical Summary}

A 64-year-old woman was referred to our department for treatment of an extensive aneurysm of the entire thoracic aorta involving the ARSA. Surgical strategy comprised 3 steps. The first step was replacement of the ascending aorta and aortic arch with the ETT and with antegrade selective cerebral perfusion. A trifurcated

From the Departments of Cardiac Surgery ${ }^{\mathrm{a}}$ and Radiology, ${ }^{\mathrm{b}}$ University of Bologna, Policlinico S.Orsola-Malpighi, Bologna, Italy.

Received for publication Sept 21, 2006; accepted for publication Oct 23, 2006.

Address for reprints: Luca Botta, MD, Cardiac Surgery Department, University of Bologna, Policlinico S.Orsola-Malpighi, Via Massarenti 9, 40138 Bologna, Italy (E-mail: allucbot@tiscali.it).

J Thorac Cardiovasc Surg 2007;133:800-1

$0022-5223 / \$ 32.00$

Copyright (C) 2007 by The American Association for Thoracic Surgery doi:10.1016/j.jtcvs.2006.10.038 prosthesis was used and a separate reimplantation of the supraortic vessels was performed. A computed tomographic scan at 1 month's follow-up demonstrated the 6-cm pre-existent aneurysm of the distal arch and of the descending thoracic aorta (DTA) and the ARSA arising from an aneurysmal dilatation of its origin, the diverticulum of Kommerell (Figure 1,a). Angiography also showed a severe stenosis of the left subclavian artery. Three months later the patient underwent a bilateral subclavian-carotid bypass as second step. Left subclavian-left common carotid artery bypass was performed to solve subclavian steal syndrome and right subclavian-right common carotid artery bypass to prepare the patient for the last step of the procedure: stent grafting of the DTA. The ARSA was ligated just proximal to the right vertebral artery. Endovascular stent-graft repair was successfully performed 1 month later. The elephant trunk was used as landing zone for the endograft. The patient had a smooth recovery and was discharged on the seventh postoperative day. A computed tomographic scan performed at 6 months' follow-up revealed a complete exclusion of the DTA aneurysm involving the origin of the ARSA (Figure 1,b).

\section{Discussion}

Surgical treatment of diffuse aneurysms of the thoracic aorta represents an exciting challenge for cardiovascular surgeons. Since the introduction of the ETT by Borst and colleagues ${ }^{3}$ in 1983, the "2-step" repair has been the standard approach for managing these aneurysms. Nevertheless, several authors have advocated single-stage repairs of the entire thoracic aorta because of the risk of death that exists while awaiting the second proce-

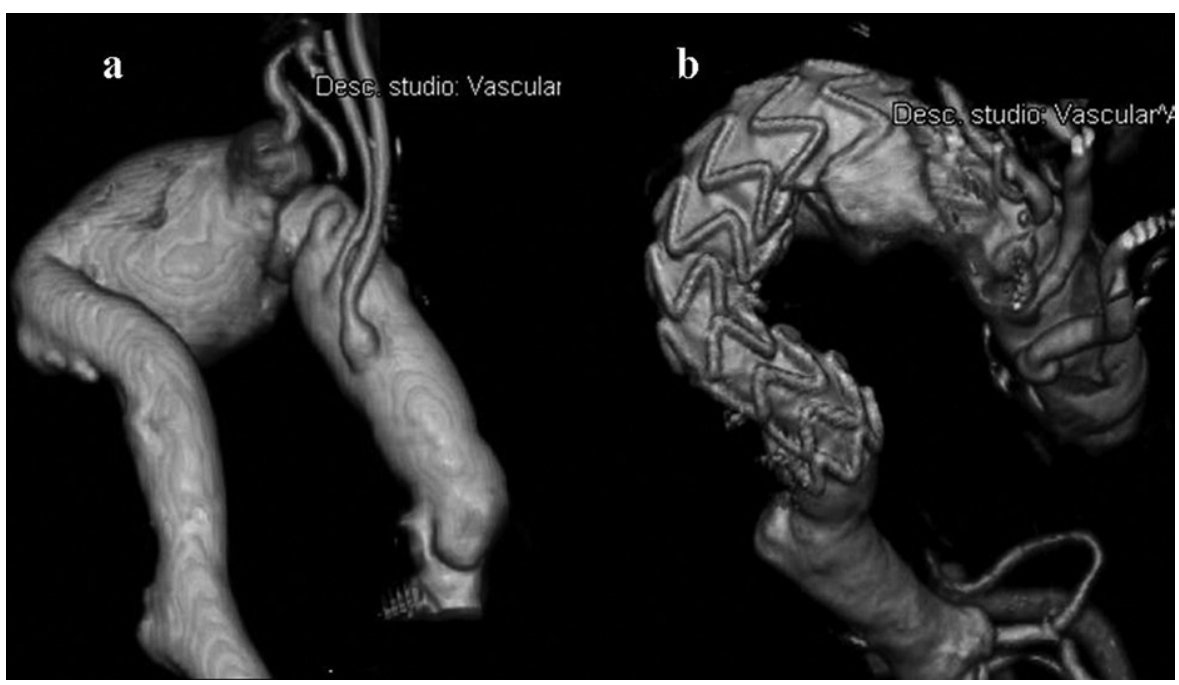

Figure 1. a, Computed tomographic scan image: Posterior view highlights the aneurysm of ARSA origin and the separate reimplantation of the supraortic vessels. b, Six-month follow-up shows an excellent surgical result with the complete exclusion of the DTA aneurysm and of the aneurysm involving the diverticulum of Kommerell. 
dure. ${ }^{1}$ The appropriate interval between operations is hard to define; LeMaire, Carter, and Coselli ${ }^{1}$ have advocated a 6-week recovery period whereas Safi and colleagues ${ }^{4}$ recommend performing the second-stage repair 4 weeks after the first stage. In patients who have had elephant trunk repair of the arch, a secondstage surgical approach via a left thoracotomy for the replacement of the DTA is usually performed. On the other hand, several groups have reported using endografts to complete the second stage of the procedure. ${ }^{1,5} \mathrm{We}$ chose endoluminal repair of the DTA aneurysm because we believed that it reduced procedural time, blood loss, length of intensive care unit stay, and hospital stay and that it is associated with lower rates of morbidity and mortality if compared with conventional open surgical procedures.

In our patient, the presence of an aneurysmal dilatation of ARSA origin made management of this already complex pathologic condition even more complicated. Most patients with ARSA are asymptomatic, but aneurysms in this location require surgical treatment since they can cause serious complications: distal embolization, rupture, and compression of neighboring structures. ${ }^{2,6}$ Because of the rareness of the ARSA aneurysms, surgical strategies are still unclear. We have performed a bilateral subclaviancarotid bypass after ETT to prepare the patient for the last step of the procedure: stent grafting of the DTA. We have modified the conventional 2-step repair of thoracic complex aneurysms adding a third stage to suit the peculiar features of our patient. The 3-stage approach has been effective and we believe that it can be safely performed in patients with diffuse aneurysms of the thoracic aorta involving an ARSA. Long-term evaluation in larger numbers of patients is necessary to verify the efficacy of this technique.

\section{References}

1. LeMaire SA, Carter SA, Coselli JS. The elephant trunk technique for staged repair of complex aneurysms of the entire thoracic aorta. Ann Thorac Surg. 2006;81:1561-9.

2. Kamiya H, Knobloch K, Lotz J, Bog A, Lichtenberg A, Hagl C, et al. Surgical treatment of aberrant right subclavian artery (arteria lusoria) aneurysm using three different methods. Ann Thorac Surg. 2006;82:187-90.

3. Borst HG, Walterbush G, Schaps D. Extensive aortic replacement using "elephant trunk" prosthesis. Thorac Cardiovasc Surg. 1983;31:37-40.

4. Safi HJ, Miller CC 3rd, Estrera AL, Huynh TT, Porat EE, Allen BS, et al. Staged repair of extensive aortic aneurysms: long-term experience with the elephant trunk technique. Ann Surg. 2004;240:677-85.

5. Greenberg RK, Haddad F, Svensson L, O'Neill S, Walker E, Lyden $\mathrm{SP}$, et al. Hybrid approaches to thoracic aortic aneurysms: the role of endovascular elephant trunk completion. Circulation. 2005;112: 2619-26

6. Austin EH, Wolfe WG. Aneurysm of aberrant subclavian artery with a review of the literature. J Vasc Surg. 1985;2:571-7.

\title{
Systemic thrombolysis for prosthetic valve thrombosis in the immediate postoperative period of major abdominal surgery
}

\author{
Manuel Ruiz-Bailén, MD, PhD, ${ }^{\mathrm{a}, \mathrm{b}}$ Benjamín Narbona-Carvo, $\mathrm{MD}^{\mathrm{c}}$ José Ángel Ramos-Cuadra, MD, ${ }^{\mathrm{d}}$ \\ Luis Rucabado-Aguilar, MD, ${ }^{a}$ Carmen López-Caler, MD, ${ }^{\mathrm{e}}$ and Francisco Javier Gómez-Jiménez, MD, Jaén, Almería, and \\ Granada, Spain
}

$\mathrm{T}$ hrombosis of a prosthetic valve is usually a cause for emergency cardiac surgery, particularly in the presence of a major contraindication for systemic thrombolysis. Recent surgery is a major contraindication for the administration of thrombolysis. Our objective is to describe the

From the Intensive Care Unit, Critical Care and Emergencies Department,

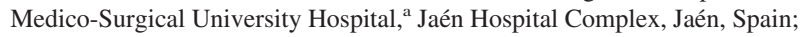
Departamento Ciencias de la Salud, Universidad de Jaén, ${ }^{b}$ Jaén, Spain; Surgery Department, Poniente Hospital, ${ }^{\mathrm{c}}$ El Ejido, Almería, Spain; Intensive Care Unit, Critical Care and Emergency Department, Torrecárdenas Hospital, ${ }^{\mathrm{d}}$ Almería, Spain; Intensive Care Unit, Critical Care and Emer-

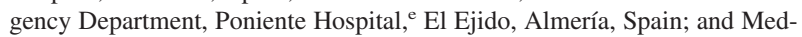
icine Department, University of Granada, ${ }^{\mathrm{f}}$ Granada, Spain.

Received for publication Sept 17, 2006; accepted for publication Oct 24, 2006.

Address for reprints: Dr. Manuel Ruiz-Bailén, C/. Las Torres 57, 23650 Torredonjimeno. Jaén, Spain (E-mail: mrb1604@teleline.es).

J Thorac Cardiovasc Surg 2007;133:801-3

$0022-5223 / \$ 32.00$

Copyright $\odot 2007$ by The American Association for Thoracic Surgery doi:10.1016/j.jtcvs.2006.10.037 administration of thrombolysis in a valve prosthesis after major surgery.

\section{Clinical Summary}

A 61-year-old male patient was admitted for lower intestinal obstruction. Sigmoidoscopy was performed and a tumor (moderately differentiated adenocarcinoma) of the upper rectum, $12 \mathrm{~cm}$ from the anal margin, was diagnosed. The patient had a metallic cardiac valve prosthesis (St Jude Medical, Inc, St Paul, Minn) in the mitral position and was being treated with acenocumarol. After reversal of the anticoagulation, the patient underwent surgery, a subtotal proctocolectomy with ileorectal anastomosis. The patient was admitted to the intensive care unit (ICU) because of hemodynamic instability in the first 24 hours, requiring high doses of dopamine and norepinephrine, with an initial diagnosis of distributive shock. Forty-eight hours after the operation, a transesophageal echocardiogram with hemodynamic assessment was performed, revealing prosthetic valve thrombosis with obstruction and abnormal disc motion of the medial hemidisc. A mean transmitral pressure gradient of $19.6 \mathrm{~mm} \mathrm{Hg}$ was observed (Figure 1). The Doppler echocardiographic hemodynamic study was compatible 